\title{
Polycystic ovaries and associated clinical and biochemical features among women with infertility in a tertiary hospital in Tanzania
}

\author{
A.B. PEMBE ${ }^{*}$ and M.S. ABEID \\ Department of Obstetrics and Gynaecology, Muhimbili University of Health and Allied Sciences, \\ P.O. Box 65117, Dar es Salaam, Tanzania
}

\begin{abstract}
The aim of this study was to determine prevalence of polycystic ovaries (PCO) and associated clinical and biochemical features among women with infertility attending gynaecological outpatient department (GOPD) at Muhimbili National Hospital (MNH) in Dar es Salaam, Tanzania. All women with infertility attending the GOPD from $11^{\text {th }}$ September 2006 to $15^{\text {th }}$ February 2007 were recruited to the study. Information on socio-demographic, obstetric and menstrual characteristics was collected. Anthropometric measurement, clinical examination of acne and hirsutism, vaginal ultrasonography for PCO and biochemical analysis of luteinizing hormone (LH), follicle stimulating hormone (FSH) and testosterone were performed. All 102 women who attended the GOPD during the study period due to infertility were recruited. Two women were excluded after diagnosis of pregnancy made by hormonal assay and ultrasonography thus remaining with 100 women for analysis. Oligomenorrhoea and acne were significantly higher in a group of women with PCO than among women with normal ovaries. The mean hirsutism score though was not significant, was higher in women with PCO than in women with normal ovaries $(5.1 \pm 2.7$ vs. $4 \pm 2.4, P<0.057)$. Using the Rotterdam criteria 32 (32\%) women were diagnosed to have polycystic ovary syndrome (PCOS). Among these women $25(78.1 \%)$ had PCO, 24 (75\%) had signs of oligoanovulation, and $18(56.3 \%)$ had hirsutism. Among 68 women with no PCOS, 7 (10.3\%) had polycystic ovaries, $15(22.1 \%)$ had signs of oligoanovulation and $6(8.8 \%)$ had hirsutism. In conclusion, polycystic ovaries are common among women with infertility, however are not necessarily associated with polycystic ovary syndrome. Doctors should investigate their clients for PCOS and offer appropriate treatment.
\end{abstract}

Key words: infertility, women, polycystic ovaries, polycystic ovarian syndrome, Tanzania

Visualization of the ovaries at laparotomy, taking a biopsy and histological examination were techniques used in the past to make diagnosis of polycystic ovaries (PCO). The advent of ultrasonography has provided a noninvasive technique for the assessment of the size and morphology of the ovaries. Prevalence of PCO varies in different studies mainly due to nature of population studied. PCO have been found in childhood, the prevalence increasing with age from $6 \%$ at 6 years of age, until $26 \%$ of the scans at 15 years of age (Bridges et al., 1993). The prevalence of PCO in normal women of reproductive age is reported to range from $14 \%$ to $33 \%$ (Polson et al., 1988; Clayton et al., 1992; Botsis et al., 1995; Michelmore et al., 1999; Koivunen et al., 1999). Taponen et al. (2004) found $47.9 \%$ of women with oligomenorrhoea only, $18.4 \%$ hirsutism only and $70.4 \%$ with both symptoms to have PCO.

The presence of PCO alone is not enough to make the diagnosis of polycystic ovarian syndrome (PCOS). The 2003 Rotterdam ESHRE / ASRM-sponsored PCOS consensus workshop group concluded that no single diagnostic criterion was sufficient for a clinical diagnosis of PCOS. It was decided that two of the three criteria had to be met to fit the definition: oligoand/or chronic anovulation (OA), clinical and/ or biochemical evidence of hyper-androgenism (HA) and PCO. Other aetiologies such as congenital adrenal hyperplasia, androgen secreting tumours, and cushings syndrome have to be excluded (ESHRE / ASRM-Workshop, 2004). The pathophysiology of PCOS is not clearly understood. However, the accumulated evidence suggests genetic predisposition and environmental factors (Dunaif \& Thomas, 2001; Legro et al., 2002).

There are no studies in Tanzania demonstrating the prevalence of polycystic ovaries among women with infertility. This cross sectional study was therefore designed to

*Dr. Andrea B. Pembe; E-mail: andreapembe@yahoo.co.uk 
determine prevalence of polycystic ovaries and its relationship with clinical and biochemical features of PCOS among women attending infertility clinic at Muhimbili National Hospital in Dar es Salaam, Tanzania.

\section{Material and Methods}

\section{Study sites and study subjects}

This descriptive study was conducted at Muhimbili National Hospital in Dar es Salaam, Tanzania from $11^{\text {th }}$ September 2006 to $15^{\text {th }}$ February 2007. All women who reported at the Gynaecological Outpatient Department (GOPD) for infertility treatment during the study period were asked to participate. Clinical assessment and laboratory investigation were carried out. Clinical assessment included age, parity, marital status, education, occupation, infertility status, menstrual pattern, family history of infertility, and duration of infertility. Menstrual pattern was characterized as regular (cycles recurring every 21-35 days) or oligomenorrhoea if menstrual cycle longer than 35 days or less than 10 menstruations per year. Infertility was defined as failure to conceive for at least one year. The infertility status was either primary (if the woman had no proven pregnancy before) or secondary (if she had proven pregnancy before).

The modified Ferrimen-Gallwey scoring method was used to assess androgen status hirsutism and acne (Ferriman \& Gallwey, 1961). It quantifies the presence of terminal hairs over nine body areas (upper lip, chin, chest, upper and lower abdomen, upper and lower back, upper arms and thighs). Acne was recorded to be present or absent by looking for comedones on the face, neck, upper chest, upper back, or upper arms. Weight, height, and waist and hip circumferences were measured. Body mass index (BMI) was calculated by dividing the weight in kilograms by the square of the height in meters.

Blood for hormonal analysis was taken irrespective of menstrual cycle. Serum samples were analyzed for levels of total testosterone, follicle stimulating hormone $(\mathrm{FSH})$ and luteinizing hormone (LH) using a double antibody technique on an Elecys 2010 analyzer (Roche Diagnostic $\mathrm{GmbH}$, Manheim, Germany) on the day blood was taken. The reagents and calibrators were supplied by the manufacturers. Transvaginal ultrasound scan (USS) was performed by an experienced physician-sonologist using Esaote Biomedica AU3 Partners, Italy and GE Logiq Pro 100, South Africa, with 7.5 MHz. USS measurements were taken in real time. The diagnosis of $\mathrm{PCO}$ was made, if 12 or more follicles, each measuring 2$9 \mathrm{~mm}$ in diameter were present in the ovarian periphery and/or ovarian volume of more than $10 \mathrm{ml}$. One polycystic ovary is sufficient for the diagnosis (ESHRE/ASRM-Workshop, 2004). The ovarian volume was estimated by measuring the diameters in three dimensions.

\section{Data analysis}

The data were entered using Epi-Info 6 then analysed by SPSS 14.0 computer programmes. Differences between two groups were evaluated with the Mann-Whitney $U$ and by Chi-square tests, as appropriate. A two-tailed P-value of $<0.05$ was considered statistically significant.

\section{Ethical considerations}

Informed consent was obtained from all women. Ethical clearance was obtained from the Senate Research and Publication Committee of Muhimbili University of Health and Allied Sciences.

\section{Results}

All 102 women who attended the GOPD during the study period due to infertility were recruited. Two women were excluded after diagnosis of pregnancy made by hormonal assay and ultrasonography thus remaining with 100 women for analysis. PCO were identified in 32 (32\%) women among 100 women included in the analysis. The baseline characteristics including age, marital status, parity and infertility status was not statistically different between women with polycystic ovaries and normal ovaries (Table 1). 
Table 1: Socio-demographic characteristics and fertility status of women with PCO and normal ovaries $(n=100)$

\begin{tabular}{lllll}
\hline Characteristics & PCO $\mathbf{n = 3 2}$ & & \multicolumn{2}{c}{ Normal ovaries $\mathbf{n}=\mathbf{6 8}$} \\
& $\mathbf{N}$ & $\mathbf{( \% )}$ & $\mathbf{N}$ & $\mathbf{( \% )}$ \\
\hline Age (years) & & & & \\
$<25$ & 6 & $(18.8)$ & 7 & $(10.3)$ \\
$25-29$ & 12 & $(37.5)$ & 16 & $(23.5)$ \\
$30-34$ & 10 & $(31.3)$ & 30 & $(44.1)$ \\
$\geq 35-$ & 4 & $(12.5)$ & 15 & $(22.1)$ \\
Marital status & & & & \\
Single & 4 & $(12.5)$ & 7 & $(10.3)$ \\
Married & 28 & $(87.5)$ & 61 & $(89.7)$ \\
Parity & & & & \\
Nulliparous & 20 & $(62.5)$ & 43 & $(63.2)$ \\
Parous & 12 & $(37.5)$ & 25 & $(36.8)$ \\
Infertility status & & & & $(60.3)$ \\
Primary & 15 & $(46.9)$ & 41 & \\
Secondary & 17 & $(53.1)$ & & \\
\hline
\end{tabular}

Menstrual pattern, clinical characteristics and biochemical markers of women with PCO and normal ovaries are presented in Table 2. Oligomenorrhoea and acne were significantly higher in a group of women with PCO than in a group of women with normal ovaries. The mean hirsutism score though was not significant, was higher in women with $\mathrm{PCO}$ than in women with normal ovaries $(5.1 \pm 2.7$ vs. $4 \pm 2.4, P<0.057)$. There was no significant difference between the groups on BMI, $25.78 \pm 5.2 \mathrm{~kg} / \mathrm{m}^{2}$ in the women with PCO and $24.16 \pm 4.0 \mathrm{~kg} / \mathrm{m}^{2}$ in women with normal ovaries $(P=0.148)$. There were no significant differences in waist-to-hip ratio, body mass index, age at menarche, levels of testosterone, $\mathrm{LH}, \mathrm{FSH}$ as well as $\mathrm{LH}$ to FSH ratio between women with PCO and normal ovaries. (Table 2)

Using the Rotterdam criteria $32(32 \%)$ women were diagnosed to have PCOS. Among these women $25(78.1 \%)$ had PCO, $24(75 \%)$ had signs of oligoanovulation, and $18(56.3 \%)$ had hirsutism. Among 68 women with no PCOS, $7(10.3 \%)$ had polycystic ovaries identified by USS, $15(22.1 \%)$ had signs of oligoanovulation and $6(8.8 \%)$ had hirsutism.

Table 2: Menstrual, clinical and hormonal profile of women with PCO and normal ovaries

\begin{tabular}{llll}
\hline Characteristic & PCO $\mathbf{n}=\mathbf{3 2}$ & Normal ovaries $\mathbf{n}=\mathbf{6 8}$ & P-value \\
\hline Age (years) & $29.3( \pm 4.5)$ & $30.9( \pm 5.1)$ & 0.153 \\
Duration of infertility (years) & $4( \pm 2.2)$ & $4.6( \pm 2.7)$ & 0.277 \\
Age at menarche (years) & $14( \pm 2.1)$ & $14.4( \pm 1.7)$ & 0.324 \\
Waist-to-hip ratio & $0.74( \pm 0.1)$ & $0.76( \pm 0.1)$ & 0.434 \\
Body mass index $\left(\mathrm{kg} / \mathrm{m}^{2}\right)$ & $25.8( \pm 5.2)$ & $24.2( \pm 4)$ & 0.090 \\
Hirsutism score & $5.1( \pm 2.7)$ & $4( \pm 2.4)$ & 0.057 \\
Testosterone $(\mathrm{ng} / \mathrm{dl})^{*}$ & $0.2(0.02-1.25)$ & $0.2(0.2-0.9)$ & 0.159 \\
LH $(\mathrm{mIU} / \mathrm{ml})^{*}$ & $7.4(0.2-72)$ & $6.5(0.3-50)$ & 0.916 \\
FSH $(\mathrm{mIU} / \mathrm{ml})^{*}$ & $5.7(0.5-44)$ & $5.2(0.4-99)$ & 0.481 \\
LH/FSH ratio & $1.7( \pm 1.2)$ & $1.7( \pm 1.8)$ & 0.867 \\
Oligomenorrhoea $(\%)$ & 53.1 & 32.4 & $<0.001$ \\
Acne $(\%)$ & 40.6 & 17.6 & 0.013 \\
\hline
\end{tabular}

${ }^{*}$ Reported as Median (Range) 


\section{Discussion}

The results of this study showed that about one-third of women attending infertility clinic were found to have PCO. A study in the United Kingdom showed a higher overall prevalence of polycystic ovaries among women with infertility was $39.4 \%$. The main specific cause was anovulatory (Kousta et al., 1999). Our study did not look into the specific causes of infertility. This prevalence is considerably higher than the reported prevalence of 17 to $23 \%$ among normal female population in a London hospital in England (Polson et al., 1988) but similar to the prevalence reported in as study in Oxford in the same country (Michelmore et al., 1999).

Oligomenorrhoea and acne were significantly more common among women with polycystic ovaries than those with normal ovaries. The results of higher prevalence of oligomenorrhoea in women with polycystic ovaries are not surprising as several studies have demonstrated similar findings. The only difference is the percentage of women with normal ovaries reported to have oligomenorrhoea $(21 \%)$ in this study is higher when compared to other studies (Clayton et al., 1992; Farquhar et al., 1994). The difference in prevalence may be due to the fact that our study population comprised of women with infertility while other studies dealt with normal women in general population.

In this study, the presence of PCO was not associated with higher levels of testosterone concentration, LH or FSH. Elevated serum testosterone concentration has been frequently associated with PCO (Balen et al., 1995). The lack of a difference in testosterone concentration between women with PCO and normal ovaries may be due to the small sample size of the study. This is further compounded by the lack of difference in the BMI between the groups. The presence of PCO has been found not to be associated with subfertility in asymptomatic women (Hassan \& Killick, 2003). Women with normal menstruation and absence of hyperandrogenism but isolated finding of $\mathrm{PCO}$ on ultrasound are not considered to have PCOS. The role of PCO in ovulating PCOS women remains unclear (Kousta et al., 1999). On the other hand, PCOS women with multiple follicles and enlarged ovarian volume have low response to ovulation induction with clomiphene citrate
(Takahashi et al., 1994).

Using the Rotterdam criteria one-third of the women had PCOS. This rate is higher compared to findings by other investigators (Knochenhauer et al., 1998; Yu Ng \& Ho, 2008). The difference observed may be due to different criteria used or the inherent difference in the prevalence of the condition in different ethnic groups (Yu Ng \& Ho, 2008). Moreover, the higher prevalence of PCOS among infertile women should be interpreted with cautions as Muhimbili National Hospital is a public tertiary referral centre, being the main provider of infertility services in the city of Dar es Salaam.

In the PCOS subgroup analysis LH was significantly higher in the group with both PCO and oligoanovulation versus the group with either oligoanovulation or PCO. This implies that combination of OA and PCO is more likely to be PCOS. Elevated values of LH have been associated with a significant impairment of fertility. It has been suggested that a high LH in the mid-follicular phase directly and adversely affects the timing of maturation of the oocyte, resulting in release of an 'aged' oocyte (Homburg et al., 1988).

This study had several limitations. It was not possible to control precisely for the time of the day of the menstrual cycle of serum sampling for $\mathrm{LH}$ and FSH as levels varies with different phases of menstrual cycle. It has been noted that none of these factors appears to have a significant impact on the clinical value of circulating androgens (Knochenhauer et al., 1998). Clinical hyperandrogenism was defined only by presence of hirsutism and oligomenorrhoea was estimated from menstrual history. Since the optimum system for acne scoring remains highly disputed (Azziz et al., 2004), its presence was simply recorded without a grade. Moreover, as total testosterone was the only androgen measured, leaving other serum androgens including androstenedione, dehydroepiandrosterone sulfate not measured. These could have lead to underestimation of the prevalence of PCOS.

In conclusion, polycystic ovaries are common among women with infertility attending a tertiary hospital in Dare s Salaam. The presence of polycystic ovaries is not necessarily associated with other symptoms of PCOS. It is important that doctors investigate their clients for PCOS and offer timely and appropriate treatment. 


\section{Acknowledgements}

Special thanks go to the research assistants Sr. Reu Mgimba and Sr. Shida Mayumana for their excellent assistance in data collection. We are grateful to Dr. Bashir Samma for the ultrasound examinations and Dr. Candida Moshiro for her advice in data analysis and interpretation.

Received 30 April 2009

Revised 10 September 2009

Accepted 12 September 2009

\section{References}

Azziz, R., Woods, K.S., Reyna, R., Key, T.J., Knochenhauer, E.S. \& Yildiz, B.O. (2004) The prevalence and features of the polycystic ovary syndrome in an unselected population. Journal of Clinical Endocrinology and Metabolism 89, 27452749.

Balen, A.H., Conway, G.S., Kaltsas, G., Techatrasak, K., Manning, P.J., West, C. \& Jacobs, H.S. (1995) Polycystic ovary syndrome: the spectrum of the disorder in 1741 patients. Human Reproduction 10, 2107-2111.

Botsis, D., Kassanos, D., Pyrgiotis, E. \& Zourlas, P.A. (1995) Sonographic incidence of polycystic ovaries in a gynecological population. Ultrasound Obstetrics and Gynecology 6, 182-185.

Bridges, N.A., Cooke,A., Healy, M.J., Hindmarsh, P.C. \& Brook, C.G. (1993) Standards for ovarian volume in childhood and puberty. Fertility and Sterility 60, 456460.

Clayton, R.N., Ogden, V., Hodgkinson, J., Worswick, L., Rodin, D.A., Dyer, S. \& Meade, T.W. (1992) How common are polycystic ovaries in normal women and what is their significance for the fertility of the population? Clinical Endocrinology (Oxf), 37, 127-134.

Dunaif, A. \& Thomas, A. (2001) Current concepts in the polycystic ovary syndrome. Annual Review of Medicine 52, 401-419.

ESHRE/ASRM-Workshop (2004) Revised 2003 consensus on diagnostic criteria and long-term health risks related to polycystic ovary syndrome (PCOS). Human Reproduction 19, 41-47.

Farquhar, C.M., Birdsall, M., Manning, P.,
Mitchell, J.M. \& France, J.T. (1994) The prevalence of polycystic ovaries on ultrasound scanning in a population of randomly selected women. Australian and New Zealand Journal of Obstetrics and Gynaecology 34, 67-72.

Ferriman, D. \& Gallwey, J.D. (1961) Clinical assessment of body hair growth in women. J Clinical Endocrinology and Metabolism 21, 1440-1447.

Hassan, M.A. \& Killick, S.R. (2003) Ultrasound diagnosis of polycystic ovaries in women who have no symptoms of polycystic ovary syndrome is not associated with subfecundity or subfertility. Fertility and Sterility 80, 966-975.

Homburg, R., Armar, N.A., Eshel, A., Adams, J. \& Jacobs, H.S. (1988) Influence of serum luteinising hormone concentrations on ovulation, conception, and early pregnancy loss in polycystic ovary syndrome. BMJ 297, 1024-1026.

Knochenhauer, E.S., Key, T.J., Kahsar-Miller, M., Waggoner, W., Boots, L.R. \& Azziz, R. (1998) Prevalence of the polycystic ovary syndrome in unselected black and white women of the southeastern United States: a prospective study. Journal of Clinical Endocrinology and Metabolism 83, 3078-3082.

Koivunen, R., Laatikainen, T., Tomas, C., Huhtaniemi, I., Tapanainen, J. \& Martikainen, H. (1999) The prevalence of polycystic ovaries in healthy women. Acta Obstetricia et Gynecologica Scandinavica 78, 137-341.

Kousta, E., White, D.M., Cela, E., Mccarthy, M.I. \& Franks, S. (1999) The prevalence of polycystic ovaries in women with infertility. Human Reproduction 14, 27202733.

Legro, R.S., Bentley-Lewis, R., Driscoll, D., Wang, S.C. \& Dunaif, A. (2002) Insulin resistance in the sisters of women with polycystic ovary syndrome: association with hyperandrogenemia rather than menstrual irregularity. Journal of Clinical Endocrinology and Metabolism 87, 21282133.

Michelmore, K.F., Balen, A.H., Dunger, D.B. \& Vessey, M.P. (1999) Polycystic ovaries and associated clinical and biochemical features in young women. Clinical Endocrinology (Oxf), 51, 779-786.

Polson, D.W., Adams, J., Wadsworth, J. \& Franks, 
S. (1988) Polycystic ovaries--a common finding in normal women. Lancet 1, 870872.

Takahashi, K., Uchida, A., Yamasaki, H., Ozaki, T. \& Kitao, M. (1994) Transvaginal ultrasonic assessment of the response to clomiphene citrate in polycystic ovarian syndrome. Fertility and Sterility 62, 48-53.

Taponen, S., Ahonkallio, S., Martikainen, H., Koivunen, R., Ruokonen, A., Sovio, U., Hartikainen, A.L., Pouta, A., Laitinen,
J., King, V., Franks, S., Mccarthy, M.I. \& Jarvelin, M.R. (2004) Prevalence of polycystic ovaries in women with selfreported symptoms of oligomenorrhoea and/or hirsutism: Northern Finland Birth Cohort 1966 Study. Human Reproduction 19, 1083-8.

$\mathrm{Yu} \mathrm{Ng}$, E.H. \& Ho, P.C. (2008) Polycystic ovary syndrome in Asian women. Seminars in Reproductive Medicine 26, 14-21. 